\title{
Practical implementation issues and challenges for biobanks in the return of individual research results
}

\author{
Marianna J. Bledsoe, MA', William E. Grizzle, MD, PhD², Brian J. Clark, MBChB, FRCPath ${ }^{3}$ \\ and Nikolajs Zeps, BSc, PhD ${ }^{4-6}$
}

Whether or not to give research results back to individuals whose specimens are used for biomedical research is a subject of considerable controversy. Much of the debate has been focused around the ethical and legal concerns with some consideration of broader social issues such as whether or not people will be affected by such information for employment or health care. Much less attention has been paid to biobanks that collect the specimens used to generate the research findings and the issues and operational requirements for implementing return of individual research results. In this article, we give the biobanks' perspective and highlight that given the diversity among the types of biobanks, it may be difficult to design and implement a blanket policy in this complex area. We discuss the variability in the types of biobanks and some important issues that should be considered in determining whether or not research results should be provided to individuals whose specimens are used in biomedical research. We also discuss challenges that should be considered in implementing any approaches to the return of research results.

Genet Med 2012:14(4):478-483

Key Words: biobanks; implementation challenges; policy; research results; return

\section{INTRODUCTION}

Human biological specimens have been collected and distributed for research purposes for many decades and have been central to advances in the understanding and treatment of human diseases. Large collections of samples have been accumulated by pathology services as part of routine practice and those affiliated with medical schools have used these archives extensively for research and teaching. In the past 40 years, population-based collections such as the Framingham ${ }^{1}$ collection have been established as well as disease-based collections such as the Cooperative Human Tissue Network ${ }^{2}$ and the National Cancer Institute Clinical Cooperative Group banks. ${ }^{3}$ More recent collections such as the UK Biobank ${ }^{4}$ have been established to meet the needs of large-scale genetic studies. Thus, the practice of using human specimens for research is not new. However, the scientific technologies for which they are used have changed over time.

Inherent in research on human specimens is the possibility of identifying new information that may be relevant to the health of the individuals from whom the specimens were obtained. The advent of increasingly affordable and rapid genome-wide association technologies has meant that discovering information about genes that are not specifically the target of individual research projects will be a routine occurrence. In addition, the number of findings with potential health relevance to individuals is expected to increase with whole-exome or whole-genome sequencing. This means that the issue of returning information to research participants must be reframed to take into account the fact that potentially relevant information will be found. Although a number of groups have issued recommendations about when research results that have potential health implications for participants should be provided, this issue remains unresolved. ${ }^{5,6}$ Furthermore, there has been much less discussion of this issue in the biobanking context.

A number of meetings have been held to address the issues of return of research results from biobanks, including a meeting sponsored by the National Cancer Institute in June 2010. ${ }^{7}$ More recently, the 2011 annual meeting of the International Society for Biological and Environmental Repositories ${ }^{8}$ brought together ethicists, biobankers, and other stakeholders for a discussion of practical implementation issues for return of individual research results from biobanks. That meeting was followed by a symposium sponsored by the University of Minnesota at which the ethical issues and draft recommendations on this topic were discussed. ${ }^{9}$

In this article, we present a series of issues that must be considered in developing guidelines for the return of individual findings from biobanks. We illustrate how these should be considered by using examples that take into account the many nuances of this very complex area, including the practical implementation issues that confront the biobanking community. These viewpoints were developed based on a review of the literature, discussions at recent meetings that included the biobanking community, and our practical experiences managing human specimen banks. 


\section{DISCUSSION}

Human specimen collections vary widely in purpose, scale, contents, and structure. Biobanks have been defined in a variety of different ways and this has been a major challenge in the field. ${ }^{10}$ When making policy recommendations for biobanks, it is essential to define the term "biobank" and to make sure that the recommendations are relevant across the broad range of biobanks that are covered within the definition. In the United States, there is no commonly accepted definition of a biobank in any policy document. For the purposes of this article, we will use the term as defined by the International Society for Biological and Environmental Repositories, "an entity that receives, stores, processes and/or distributes specimens, as needed" ${ }^{11}$ and limit our discussion to biobanks of human specimens and associated data established for research purposes.

Although there appears to be a clear difference in terms of original intent between information that arises from the direct purpose of the research and data that emerge serendipitously, many of the ethical considerations, as well as practical implementation issues are similar. Thus, for the purpose of this article, we will treat individual research results and incidental findings as the same.

\section{Ethical issues and research participant's perspectives}

There has been considerable debate about whether, when, and how research results should be provided back to research participants or their physicians. Some have made arguments for the return of individual research findings that include respect for persons, beneficence, reciprocity, justice, and the duty to rescue..$^{12,13}$ Others have argued against the return of research results on several grounds including the original intent being an altruistic donation to help research. ${ }^{14}$ Additional concerns with a general framework of giving back individual results include the important view that it promotes a therapeutic misconception (the conflation of research and clinical care) and that harms can accrue when individual research findings that have not been validated are returned to participants or their physicians. ${ }^{15}$ Although the ethical principle of beneficence has been interpreted by some as a need to ensure some benefit to the individual for participation, ${ }^{16}$ this concept in itself raises concerns about incentives for participation and the possibility of undue inducement. Some studies indicate that when asked, research participants say that they are interested in receiving individual research results. ${ }^{17-19}$ As others have noted, this finding must be treated with caution as survey responses may not actually reflect what participant's preferences would be if they were given more information about the limitations in the meaning of individual research results. ${ }^{13}$ In addition, research participants' views may vary depending on the context; for example, whether the research participant is a patient or a member of the general population. ${ }^{20}$ Other studies suggest that although there is a desire to receive results, some research participants may be concerned that the resource requirements of providing individual results to participants could detract from the research itself. ${ }^{21}$ Furthermore, some have argued that the participants' desire to receive individual research results does not necessarily mean that individual research results should be offered to them..$^{22}$

\section{Variability in biobanks}

Biobanks can vary in scale from individual collections of just a few to many thousands of cases. Biobanks can be project specific or they may collect specimens for secondary research projects. They may obtain samples from healthy persons or from those with disease and may be used for a single type of research (e.g., cancer or genomic research) or a wide variety of types of research. They may be organized and operated in a variety of different ways: for example, as a prospective tissue collection system, where specimens and data are collected and rapidly distributed to meet individual researcher's needs; as a centralized tissue bank, where specimens and data are collected from multiple sites and the data and specimens stored centrally; or a virtual bank, where specimens and data are collected at multiple sites, but the specimens and data remain at the sites at which they are collected until a specific research use has been identified. The practical implementation of return of individual research results will vary enormously as a result of the wide variability in biobank types. Below, we discuss some of these practical implementation issues and variability based on the type of biobank with some examples.

\section{Practical implementation issues}

Privacy and confidentiality. The return of individual findings to research participants requires that biobanks retain links to identifying information about them. Although some biobanks must retain links to identifying information to be able to follow participants over time, others do not need to retain such information to meet their intended research purpose. Retaining links to identifiers for the purpose of recontact to provide individual research results and ensuring that the results are specific to an individual participant raises risks to privacy of participants and the confidentiality of their data.

Transfer of samples, tracking, and labeling. Because of potential harms to participants, there must be procedures in place to ensure that the sample tested actually came from the person it is believed to have come from. Biobanks have quality-assurance procedures in place to ensure that the samples are accurately labeled. However, to protect the privacy of the participants and the confidentiality of their data, samples are often re-coded before they are distributed to researchers. Similarly, although researchers will take steps to ensure that the results from individual samples are properly ascribed, additional opportunities for error in individual findings may be introduced. In clinical labs, several pieces of identification, such as initials, date of birth, laboratory number, and episode number, are used to cross-check identity. However, in the biobank and research laboratory typically only a code is used as a result of the intent to maximize confidentiality. With more persons involved and reduced identity cross-checks it is statistically more probable that more errors are made than occur in a clinical laboratory. Such mistakes are estimated to 
occur with up to $0.1 \%$ frequency in a diagnostic laboratory. ${ }^{23}$ In a research setting where the focus is on generating findings for populations and not individual findings for the purpose of health care and specimens may pass through many hands, the likelihood of error may be higher. It is, therefore, essential that any test result from a research laboratory be repeated on either a fresh sample or an archived sample from the original specimen.

Quality assurance and control. Sample degradation is widely seen to be a significant issue for using specimens and great pains are taken to ensure that this is minimized. Perhaps more important, it is essential to know that the sample used for research is accurately characterized. This is particularly the case with the use of human cancer specimens due to the nature of how they are obtained in pathology and the fact that often the sample can have very low actual tumor content. This issue has been reported widely with proposals for rigorous quality control in collection, storage, and processing. ${ }^{24,25}$ Even with such quality control, diseased cells may not be separated from nondiseased cells in assays resulting in false-negatives or falsepositives when using techniques such as Sanger sequencing.

Even if the samples are of appropriate quality, it is essential that tests are performed to a clinical standard using approved methodologies. Because of concerns about quality control of individual research results, regulations such as those of the Clinical Laboratory Improvements Amendments ${ }^{26}$ in the United States, require that only certified/accredited laboratories can provide test results that can go back to patients via their physicians. Almost no research laboratories and/or research biobanks have such standards and few will have the significant resources required to get such accreditation.

Validity of the findings. In addition to the need to be analytically valid, some groups have recommended that individual research results only be returned if they are clinically valid. ${ }^{27,28}$ (For the purposes of this article, we use the following definitions adapted from those of the Secretary's Advisory Committee on Genetics, Health and Society. Analytical validity is the ability of a test to measure a particular characteristic (e.g., a DNA sequence) accurately and reliably in a given specimen. Clinical validity refers to the test's accuracy in detecting the presence of, or predicting the risk for, a health condition or phenotype (see http://www.ncbi.nlm.nih.gov/pmc/articles/PMC2873211/). In many instances, it is not clear what the results may actually mean for an individual. Although the number of genetic markers that prove to be analytically and clinically valid may increase with time, there are at present very few validated genetic risk factors.

Concerns have also been expressed over the rush of some researchers to make claims for their testing that have not been substantiated in sufficiently large trials to assure clinical validity. In one example that has led to legal action, it was claimed that an experimental test for cancer was used on patients resulting in operations that may not have been necessary. ${ }^{29}$ In a more recent example, some clinical studies were based on genetic studies that were demonstrated to be invalid and this required their closure. ${ }^{30}$ Even if outside panels of experts are established to determine when tests have been sufficiently validated to be returned to participants, as some have suggested ${ }^{6,7}$ resources are required to set up such structures. Furthermore, by the time results are sufficiently validated to be returned to participants, they may be irrelevant in some cases. These and other practicability issues are discussed further below.

Legal liability. As noted previously by others, ${ }^{31}$ return of individual research results and incidental findings raises liability issues. This issue is particularly complex in the case of biobanks where multiple entities are involved with the collection, storage, distribution, and use of the specimens. In such cases, who will be responsible for damages resulting from the return of incorrect results or inappropriate use of invalid results? In the case of biobanks where the research findings are generated by a secondary researcher, the biobank usually has no control over the quality of the data. Biobankers will not want to be liable for such findings and, as such, may be unwilling to participate in biobanking if return of individual research results in such cases is required.

Practicability, infrastructure requirements, and costs. In addition to ethical considerations regarding return of individual research results, there are also practicability and cost considerations. If one accepted that in some instances research results could be fed back, assuming all of the above considerations had been addressed, how practicable is such an undertaking? Some biobanks distribute large numbers of specimens and data per year to many investigators. For example, the Cooperative Human Tissue Network distributes $>40,000$ specimens per year to hundreds of researchers (unpublished data). The impact of returning research results on biobanks that distribute large numbers of specimens and data could be significant. Setting up systems to return individual research results has infrastructure implications and costs, including the need to set up systems for decision-making and processes for implementing the return of findings, staffing and funding for recontact, informatics systems for reporting and auditing and tracking of specimens, etc. In addition, as the return of clinically important information should generally be provided with the involvement of a medical professional, biobanks returning individual results would need to establish relationships with and hire medical professionals, genetic counselors, and other personnel. There are also issues and infrastructure costs related to training and education of researchers, biobanking staff, research coordinators and physicians, research participants, and the public. In many cases, it would be impracticable to set up such systems for widespread return of results at the present funding levels of biobanks and it would significantly expand the scope of biobank responsibilities. In the case where return of medically relevant research results is warranted and feasible, additional funding to support such efforts will need to be provided.

One recent genetic epidemiology study reported the time and costs of disclosing individual research results to research participants. ${ }^{32}$ In this study, melanoma survivors were tested for CDKN2A as a marker for increased risk of melanoma. A subset 
of 39 of the total participants $(N=663)$ were approached for the return of results; of these, 27 were successfully contacted, and 19 sought results. Costs for returning results from this subset of participants, which included the costs of retesting archived DNA samples, genetic counseling, recontact of participants, and follow-up, were estimated at $\$ 1,322$ per completed disclosure. The disclosures in this study were for the results of tests for a single gene mutation. The costs for disclosures are likely to be much higher for biobanks where the research findings are not limited to a single mutation, but include a variety of different types of research findings or alterations across the entire genome because of the need to tailor education and counseling to the specific research tests being evaluated. In the case of biobanks that distribute large numbers of specimens for research, the costs could be prohibitive.

In addition to the cost, the temporal limitations to recontacting participants must be considered. In many cases, the samples may have been collected many years previously and the return of individual research results in such cases is infeasible as it may be difficult to locate the individuals from whom the specimens were obtained. Maintaining an up-to-date database to follow subjects for the sole purposes of recontact to provide individual research results may be costly and impractical in some cases.

\section{Context matters}

As noted by others, ${ }^{19}$ context really matters when considering whether to offer individual research results to participants, and some of these variables are shown in Table 1. A major consideration when deciding whether to give back individual results relates to the relationship the biobank has with the individuals from whom the specimens are obtained. In some cases, the biobank may have an ongoing relationship with the individuals, such as in prospective, longitudinal cohort studies. In other cases, the biobank has no relationship with the individuals from whom the specimens were obtained. When there is no relationship, returning individual research results is problematic. As illustrated in the following examples, the nature of the relationship and practical implementation issues regarding the return of research results to participants will vary according to the design of the biobank.

\section{Examples}

Prospective tissue distribution system. Some biobanks, such as the Cooperative Human Tissue Network, ${ }^{2}$ procure residual specimens collected during the course of routine care from multiple collecting sites to meet individual investigator's research needs. The biobank has no relationship with the patients from whom the samples are obtained. Tens of thousands of specimens are distributed to hundreds of researchers each year for basic and developmental studies. Given the nature of these biobanks, the expectation for clinically relevant research or incidental findings would be quite small, with the exception being possible discordant pathologic diagnoses. When there is discordant pathologic diagnostic information, this is typically provided to the original diagnostic pathologist as part of quality control.
Table 1 Variables in biobanking that have practical implications for the return of individual research findings

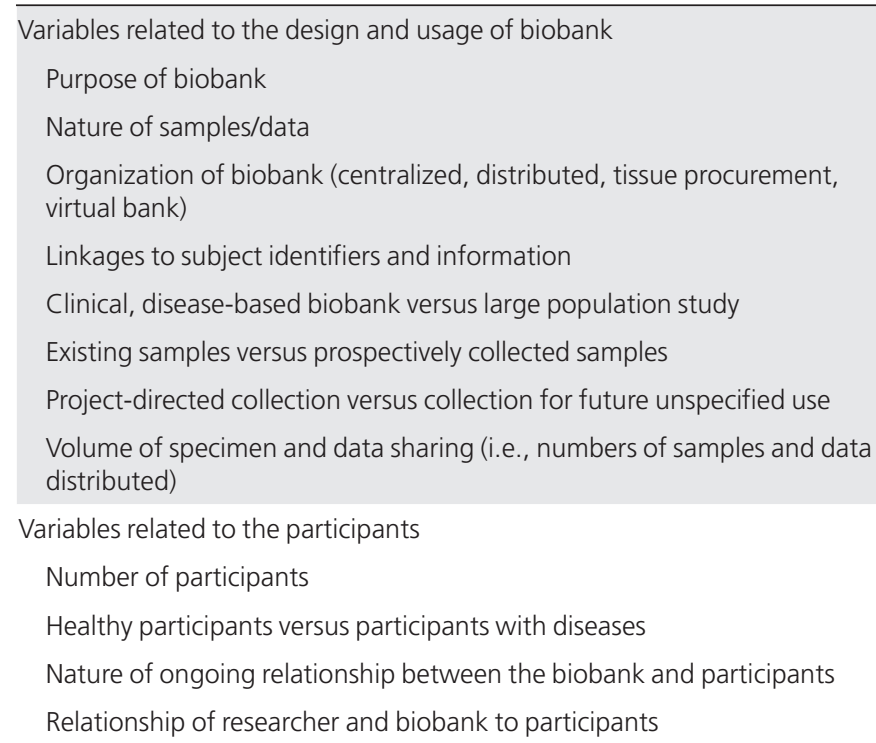

Extent of prior consent: none, consent silent on return of results, consent addresses return of results

Ease and possibility of recontact of participants

Variables related to the research

Likelihood that clinically relevant findings will be generated

Clinical relevance/significance of expected results

Accuracy

Clinical reliability

Interpretation of results

Time between specimen collection, subject consent, and generation of research finding

Because the biobank has only a small sample of the specimen, the original diagnosis may be based on other heterogeneous areas of the tissue as well as supporting molecular assays. The treating physician has no formal role in the biobank and no involvement in the research performed with the specimens. In addition, quality control and sample-tracking issues are important issues. In many cases, the patient will have already been treated by the time the research using the samples is completed and the individual research result is validated. Thus, it would not be reasonable to feed back individual research results from these banks as a general matter.

Diseased-based external biobank. In many instances, the biobank acts as an external party responsible for sample collection that is separated from patient care. One such example is the Australian Prostate Cancer Bio-Resource. ${ }^{33}$ In such cases, treating physicians have no formal role in the biobank and no enduring association that will enable feedback. In addition, if the biobank was established using specimens from pathology archives, returning clinically relevant data raises practicability issues such as how to provide the proper medical framework for doing so, privacy concerns, and legal and ethical issues. ${ }^{34}$ There 
may have been no explicit consent for research as informed consent may have been waived by an institutional review board or ethics review committee. In many cases, the samples may have been collected years ago. The return of individual research results in such cases is infeasible as it may be difficult to locate the patients from whom the specimens were obtained. In addition, there are quality-control and sample-tracking issues resulting from secondary research uses, and if many years have passed, the individual research result, even if analytically and clinically valid, may no longer be relevant to the individual's health. Furthermore, and perhaps most important, the return of individual results to individuals who may not be aware that their specimens have been used in research is highly problematic from an ethical standpoint. Recontacting them to ask them whether they consent to recontact to receive research results may be an unwelcome intrusion. ${ }^{35}$ These biobanks should not be responsible for managing return of individual research results.

Local, prospective, longitudinal cohort biobank. Locally collected samples obtained in prospective, longitudinal cohort studies represent another type of biobank. In these studies, consent for sample collection and use is obtained prospectively, often with a direct and ongoing relationship between the investigator/ biobanker and the participant. The investigator/biobanker uses the samples for his/her own research. In these cases, it may be reasonable to consider, under certain limited circumstances, the return of individual research findings from validated tests because participants have consented, there is a direct and ongoing relationship with the participant, and there is greater control of the specimen tracking. However, concerns about quality assurance and control, validity of the findings, and the need to independently validate an individual result in a Clinical Laboratory Improvements Amendments-approved laboratory still pertain and general research results may not be applicable to all ethnic and racial subpopulations. In addition, it is not clear who would be responsible for paying for such testing.

These examples demonstrate how the variability in types of biobanks affects their ability to return individual research results to participants and the practical implementation issues that must be considered in designing policies regarding the return of individual research findings.

\section{SUMMARY AND RECOMMENDATIONS}

For the reasons discussed previously, we believe that great caution must be exercised with regard to establishing blanket policies regarding the return of research results from biobanks. Errors in sample tracking and labeling as the result of transfer of samples to multiple parties and quality assurance and control issues in the biobanking context may raise the likelihood of errors in individual findings. Attention must be paid to the very real risk of harm to participants if incorrect results or results that have been misinterpreted are returned, especially if the proper infrastructure to ensure that the information is understood and used appropriately is not also provided.
In addition, liability and practical implementation issues and requirements may discourage or hinder the development of biobanks and make it difficult for some to continue to operate. With scarce funding and very limited resources of biobanks, other activities, issues, and requirements to do anything else besides providing tissues reduces the ability of a biobank to support research and creates delays in answering critical research questions that can lead to new treatments and improvements in public health. This results in a cost to society of inhibiting important research needed to improve the health of individuals and the public. In addition, returning findings with uncertain clinical significance may place additional burdens on physicians who may not be prepared to discuss the results with participants who receive them and may result in additional testing and further demands on already strained health-care systems. Others have previously noted that when deciding whether to provide individual research results, it is essential to assess not only the risks and benefits to individuals but also the risks and benefits to the research enterprise and society as a whole. ${ }^{36}$

Biobanks need policies and plans upfront for determining if, when, and how results will be returned..$^{37}$ These plans should be clearly articulated in the protocol and, where relevant, in the informed consent process. However, the wide variability in biobanks makes it difficult to establish a "one-size-fits-all" approach. Rather than prescribing policies for return of individual research results that would be intended to apply across all types of biobanks, we suggest an approach similar to that recommended by the Australian National Statement on Ethical Conduct in Human Research, where biobankers must submit an ethically defensible plan to their ethics committee for when, if ever, and how to return individual research results and that return of individual research results be considered on a case-by-case basis based on an evaluation of the risks and benefits to individuals and costs to society. ${ }^{38}$ Biobanks serve a public good, and unduly burdening biobanks with requirements for returning individual research results comes at a cost to society. Rather than returning individual results that may not be accurate or have unclear clinical meaning, biobanks can show respect for participants by sharing lists of published papers or other summary information about the research made possible by the biobank where appropriate through newsletters, websites, and other media. ${ }^{39,40}$ The costs associated with setting up elaborate infrastructures to return individual results from biobanks may best be utilized for additional research that will advance the health of patients and the public.

\section{ACKNOWLEDGMENTS}

This work was supported in part by the Department of Veterans Affairs, Veterans Health Administration, Office of Research and Development, Biomedical Laboratory (M.J.B.), St John of God HealthCare (N.Z.) and the following grants to W.E.G.: 5U01CA44968, 5P50CA89019, 2P50CA101955, P30CA-13148, and 5P50CA098252. Presentations at conferences and discussions contributing to the content of this article were supported in part by the International Society for Biological and Environmental Repositories and the National Institutes of Health, National 
Human Genome Research Institute grant no. 2-R01-HG003178 on "Managing Incidental Findings and Research Results in Genomic Biobanks \& Archives (S. Wolf, principal investigator).

\section{DISCLOSURE}

M.J.B. is a program manager for the Department of Veteran's Affairs biorepositories and biobanks and is a member of the International Society for Biological and Environmental Repositories (ISBER). The viewpoints expressed herein represent the author's personal perspectives and should not be taken as representing the official viewpoint or position of the Department of Veterans Affairs. N.Z. operates a cancer biobank at St John of God HealthCare within its Pathology Practice in the Bendat Family Comprehensive Cancer Centre. He is a member of ISBER and the Australasian Biospecimen Network Association. W.E.G. operates both tumor banks as part of the Breast, Pancreatic and Cervical SPOREs at the University of Alabama at Birmingham and the Pulmonary Hypertension Breakthrough Initiative and prospective tissue repositories as part of the Cooperative Human Tissue Network and the Comprehensive Cancer Center and is a member of ISBER. B.J.C. manages a national research biobank at Qatar Foundation and is a member of ISBER.

\section{REFERENCES}

1. National Heart, Lung and Blood Institute and Boston University. Framingham Heart Study. http://www. framinghamheartstudy.org/. Accessed 30 November 2011.

2. National Cancer Institute. Cooperative Human Tissue Network. http://chtn. nci.nih.gov/. Accessed 30 November 2011.

3. Leyland-Jones BR, Ambrosone CB, Bartlett J, et al.; Breast International Group; Cooperative Groups of the Breast Cancer Intergroup of North America (TBCI); American College of Surgeons Oncology Group; Cancer and Leukemia Group B; Eastern Cooperative Oncology Group; North Central Cancer Treatment Group; National Cancer Institute of Canada Clinical Trials Group; Southwest Oncology Group; National Surgical Adjuvant Breast and Bowel Project; Radiation Oncology Group; Gynecologic Oncology Group; Children's Oncology Group. Recommendations for collection and handling of specimens from group breast cancer clinical trials. J Clin Oncol 2008;26:5638-5644.

4. Ollier W, Sprosen T, Peakman T. UK Biobank: from concept to reality. Pharmacogenomics 2005;6:639-646.

5. Wolf SM, Lawrenz FP, Nelson CA, et al. Managing incidental findings in human subjects research: analysis and recommendations. J Law Med Ethics 2008:36:219-248, 211.

6. Fabsitz RR, McGuire A, Sharp RR, et al.; National Heart, Lung, and Blood Institute working group. Ethical and practical guidelines for reporting genetic research results to study participants: updated guidelines from a National Heart, Lung, and Blood Institute working group. Circ Cardiovasc Genet 2010;3:574-580.

7. National Cancer Institute Office of Biorepositories and Biospecimen Research. NCI Workshop on Release of Research Results to Participants in Biospecimen Studies. http://biospecimens.cancer.gov/resources/publications/workshop/rrra.asp. Accessed 30 November 2011.

8. ISBER. ISBER 2011 Annual Meeting \& Exhibits. http://www.isber.org/ mtgs/2011/program.html. Accessed 30 November 2011.

9. University of Minnesota. "Should We Return Individual Research Results and Incidental Findings from Genomic Biobanks \& Archives?" http://www. lifesci.consortium.umn.edu/conferences/2011_ifbb/agenda. Accessed 30 November 2011.

10. Kaye J. Do we need a uniform regulatory system for biobanks across Europe? Eur J Hum Genet 2006;14:245-248.

11. ISBER best practices for repositories: collection storage, retrieval, and distribution of biological materials for research. Cell Preserv Technol 2008;6:5-58.
12. Ravitsky $\mathrm{V}$, Wilfond $B S$. Disclosing individual genetic results to research participants. Am J Bioeth 2006;6:8-17.

13. Beskow LM, Burke W. Offering individual genetic research results: context matters. Science Translational Medicine 2010;2(38):1-5.

14. Forsberg JS, Hansson MG, Eriksson S. Changing perspectives in biobank research: from individual rights to concerns about public health regarding the return of results. Eur J Hum Gen 2009;17:1544-1549.

15. Clayton EW, Ross LF. Implications of disclosing individual results of clinical research. JAMA 2006;295:37; author reply 37-38.

16. Allen C, Foulkes WD. Qualitative thematic analysis of consent forms used in cancer genome sequencing. BMC Med Ethics 2011;12:14.

17. Murphy J, Scott J, Kaufman D, Geller G, LeRoy L, Hudson K. Public expectations for return of results from large-cohort genetic research. Am J Bioeth 2008;8:36-43.

18. O'Daniel J, Haga SB. Public perspectives on returning genetics and genomics research results. Public Health Genomics 2011:14;346-355.

19. Beskow LM, Smolek SJ. Prospective biorepository participants' perspectives on access to research results. J Empir Res Hum Res Ethics 2009;4:99-111.

20. Bovenberg J, Meulenkamp T, Smets E, Gevers S. Biobank research: reporting results to individual participants. Eur J Health Law 2009;16:229-247.

21. Kaphingst KA, Janoff JM, Harris LN, Emmons KM. Views of female breast cancer patients who donated biologic samples regarding storage and use of samples for genetic research. Clin Genet 2006;69:393-398.

22. Meulenkamp TM, Gevers SK, Bovenberg JA, Koppelman GH, van Hylckama Vlieg A, Smets EM. Communication of biobanks' research results: what do (potential) participants want? Am J Med Genet A 2010;152A:2482-2492.

23. Wagar EA, Stankovic AK, Raab S, Nakhleh RE, Walsh MK. Specimen labeling errors: a Q-probes analysis of 147 clinical laboratories. Arch Pathol Lab Med 2008;132:1617-1622

24. Grizzle WE, Sexton KC, Bell WC. Quality Assurance in Tissue Resources Supporting Biomedical Research. Cell Preserv Technol 2008;6:113-118.

25. Moore HM, Kelly A, Jewell SD, et al. Biospecimen Reporting for Improved Study Quality. Biopreserv Biobank 2011;9:57-70.

26. Centers for Disease Control and Prevention (CDC). Current CLIA Regulations. http://wwwn.cdc.gov/clia/regs/toc.aspx. Accessed 30 November 2011.

27. National Bioethics Advisory Commission. Research Involving Human Biological Materials: Ethical Issues and Policy Guidance (1999). http:// bioethics.georgetown.edu/nbac/pubs.html. Accessed 30 November 2011.

28. Caulfield T, McGuire AL, Cho M, et al. Research ethics recommendations for whole-genome research: consensus statement. PLoS Bio/ 2008;6:e73.

29. Gosselin PG. Duke settles misdiagnosis in cancer case. Boston Globe 5 October 1989, p 89.

30. Reich ES. Missing part delays space mission. Nature 2011;469:280.

31. Dressler LG. Biobanking and disclosure of research results: addressing the tension between professional boundaries and moral intuition. In: Solbakk JH, et al. (eds). The Ethics of Research Biobanking. Springer: New York, 2009;85-99.

32. Christensen KD, Roberts JS, Shalowitz DI, et al. Disclosing individual CDKN2A research results to melanoma survivors: interest, impact, and demands on researchers. Cancer Epidemiol Biomarkers Prev 2011;20:522-529.

33. National Health and Medical Research Council. Australian Prostate Cancer Bio-Resource. http://www.apccbioresource.org.au/. Accessed 30 November 2011.

34. Zeps N, lacopetta BJ, Schofield L, George JM, Goldblatt J. Waiver of individual patient consent in research: when do potential benefits to the community outweigh private rights? Med J Aust 2007;186:88-90.

35. Clayton EM. Incidental findings in genetics research using archived DNA. J Law Med Ethics 2008;286-291.

36. Beskow LM. Considering the nature of individual research results. Am J Bioeth 2006;6:38-40; author reply W10-12.

37. Haga SB, Beskow LM. Ethical, legal, and social implications of biobanks for genetics research. Adv Genet 2008;60:505-544.

38. Australian Government National Health and Medical Research Council. National Statement on Ethical Conduct in Human Research 2007 - Updated 2009. http://www.nhmrc.gov.au/guidelines/publications/e72. Accessed 30 November 2011.

39. Beskow LM, Burke W, Merz JF, et al. Informed consent for population-based research involving genetics. JAMA 2001;286:2315-2321.

40. Shalowitz DI, Miller FG. Disclosing individual results of clinical research: implications of respect for participants. JAMA 2005;294:737-740. 